\title{
Habilidade competitiva relativa de arroz irrigado com arroz-vermelho suscetível ou resistente ao herbicida imazapyr + imazapic
}

\author{
Relative competitive ability of irrigated rice with red rice susceptible or \\ resistant to the herbicide imazapyr + imazapic
}

\section{Rafael Salles Rubin ${ }^{1 *}$, Ana Claudia Langaro', Franciele Mariani ${ }^{1}$, Dirceu Agostinetto', Roberta Manica Berto'}

| | | | | | | | | | | | | | | | | | | | | | | | | | | | | | | | | | | | | | | | | | | | | | | | | | | | | | | | | | | | | | | | | | | | | | | | | | | | | | | | | | | | | | | | | | | | | | | | | | | | | | | | | | | | | | | | | | | | | | | | | | | | | | | | | | | | | | | | | | | | | | | | | | | | | | | | | | | | | | | | | | | | | | | | | | | | | | | | | | | | | | | | | | | | | | | | | |

\begin{abstract}
RESUMO: O uso intenso de herbicidas com o mesmo mecanismo de ação tem selecionado espécies de plantas daninhas resistentes como, por exemplo, o arroz-vermelho (Oryza sativa L.) com resistência à mistura formulada dos herbicidas imazapyr + imazapic. A competiçáo de plantas daninhas ocasiona perda de produtividade das culturas, geralmente crescente, quanto mais semelhantes forem suas características morfofisiológicas. Ainda, a capacidade competitiva do biótipo geralmente é alterada pela característica de resistência, e seu conhecimento é fundamental para a compreensão da dinâmica da população resistente. Diante disso, o objetivo da pesquisa foi estudar a habilidade competitiva entre biótipos de arroz-vermelho resistente e suscetível ao herbicida imazapyr + imazapic com arroz irrigado, utilizando o método de série de substituição. Para isso, foram conduzidos experimentos com a cultivar Puitá INTA CL, competindo com o biótipo de arroz-vermelho suscetível ou resistente. As populaçóes de plantas por vaso totalizaram 24, o equivalente a 942 plantas $\mathrm{m}^{-2}$. Para o arroz-vermelho suscetível ou resistente ao herbicida, a competição interespecífica é favorável em relação à competição intraespecífica, enquanto para a cultura do arroz irrigado o competidor independe. A cultivar Puitá INTA CL, em geral, apresenta habilidade competitiva equivalente aos biótipos de arroz-vermelho suscetível ou resistente ao herbicida imazapyr + imazapic, competindo pelos mesmos recursos do ambiente.
\end{abstract}

PALAVRAS-CHAVE: Oryza sativa; competição; imidazolinonas; resistência.

\begin{abstract}
The intensive use of herbicides with the same mechanism of action has selected resistant weeds, among which is the red rice (Oryza sativa L.) after the use of the formulated herbicide mixture of imazapyr and imazapic. The competition of weeds leads to usually increasing yield losses of crops, the more similar the morphological and physiological characteristics between weed and crop. Moreover, the competitive ability of the biotype is generally modified by the resistance characteristic; besides, the knowledge of competitive ability is fundamental to understand the dynamics of the resistant population. Therefore, the aim of this research was to study the competitive ability of red rice biotypes that are resistant and susceptible to imazapyr and imazapic herbicide with rice, using the series substitution method. Experiments were conducted with Puitá INTA CL cultivar competing with susceptible or resistant biotypes of red rice. The total plant populations per pot were of 24 , similar to 942 plants $\mathrm{m}^{-2}$. For red rice that is resistant or susceptible to herbicide, the interspecific competition is favorable with regard to intraspecific competition, while for the irrigated rice the competitor is independent. Generally, the Puitá INTA CL cultivar presents competitive ability equivalent to red rice biotypes that are susceptible or resistant to imazapyr and imazapic herbicide, competing for the same resources from the environment.
\end{abstract}

KEYWORDS: Oryza sativa; competition; imidazolinone; resistance. 


\section{INTRODUÇÃO}

$\mathrm{O}$ arroz vem se destacando como uma das culturas mais importantes no sul do Brasil. A produtividade média do arroz no Rio Grande do Sul (RS) e em Santa Catarina (SC) cresceu nos últimos anos, atingindo, na última safra, 7,6 $\mathrm{t} \mathrm{ha}^{-1}$ (ConAB, 2011), reflexo, especialmente, da utilização de cultivares com alto potencial produtivo e da adoção de novas tecnologias. Porém, esta produtividade ainda está aquém daquela alcançada em áreas experimentais, o que decorre principalmente do controle insatisfatório de plantas daninhas, as quais causam prejuízos quantitativos e qualitativos na produção do cereal; por isso, a adoção de medidas de controle é indispensável (Fleck et al., 2008).

A utilização de herbicidas para o controle das plantas daninhas, aliada ao desenvolvimento de novas tecnologias de aplicação, representou avanço na agricultura. $\mathrm{O}$ controle químico de plantas daninhas através de herbicidas justifica-se por reduzir a competição desde o início do ciclo da cultura, controlar as infestantes em épocas chuvosas, causar poucos danos às raízes e folhas das culturas, além de gerar rapidez de utilização e baixo custo quando comparado a outros métodos de controle (Vidal; Merotto JR., 2001).

$\mathrm{Na}$ cultura do arroz irrigado, a competição com plantas daninhas, se não forem controladas, pode acarretar perdas na produçáo de aproximadamente $85 \%$ (FLECK et al., 2004). Dentre as plantas daninhas que infestam a lavoura de arroz destaca-se o arroz-vermelho (Oryza sativa) (AgostinetTo et al., 2001), principalmente pela grande dificuldade de controle, uma vez que pertence à mesma espécie do arroz cultivado (Oryza sativa) (FISCHER; RAMirez, 1993). Isso limita muito o uso de herbicidas, pois são poucos os que são seletivos para a cultura.

Plantas daninhas estão em constante evolução, adaptando-se a perturbaçóes provocadas pela natureza e pelo homem (RADOSEVICH et al., 1997). Assim, aplicaçóes constantes de herbicidas têm proporcionado seleção da flora, podendo resultar em populações resistentes (Christoffoleti et al., 2008), o que é indesejável.

Um aspecto importante é compreender as mudanças que ocorrem na planta associada com a resistência. Dessa forma, obter conhecimento sobre a capacidade competitiva do biótipo resistente, a qual pode ser alterada pela característica de resistência, é fator fundamental para a compreensão da dinâmica da população resistente (VILA-Aıub et al., 2009).

Um estudo realizado para avaliar a habilidade competitiva entre biótipo de Cyperus difformis resistente ou suscetível a herbicidas inibidores da enzima acetolactato sintase (ALS), e destes com arroz irrigado, demonstrou que os biótipos apresentam habilidade competitiva equivalente (DAL MAGro et al., 2011). Ainda, os autores observaram que a cultura do arroz mostrou habilidade competitiva superior à dos biótipos. Porém, foram observados resultados nos quais as plantas daninhas capim arroz (Echinochloa sp.) e arroz-vermelho
(Oryza sativa) foram mais competitivas que a cultura do arroz (Agostinetto et al., 2008; Fleck et al., 2008).

As interaçóes competitivas entre plantas daninhas e culturas podem ser quantificadas por vários métodos experimentais, com destaque para o em série de substituição, o qual possibilita o estudo da competição inter e intraespecífica e permite indicar qual espécie é a mais competitiva (Cousens, 1991). A premissa desse tipo de experimento é de que as produtividades das associaçóes possam ser determinadas em comparação às do monocultivo, mantendo constante a população total de plantas.

Assim, o objetivo da pesquisa foi estudar a habilidade competitiva entre biótipos de arroz-vermelho resistente e suscetível ao herbicida imazapyr + imazapic com arroz irrigado, utilizando o método de série de substituição.

\section{MATERIAL E MÉTODOS}

Foram conduzidos dois experimentos em casa de vegetação, na Faculdade de Agronomia Eliseu Maciel (FAEM) da Universidade Federal de Pelotas (UFPel), no município de Capão do Leão (RS), na estação de cultivo 2010/2011. O delineamento experimental foi inteiramente casualizado, com quatro repetiçôes. Os ensaios foram alocados em vasos plásticos com capacidade volumétrica de $6 \mathrm{~L}$, preenchidos com solo oriundo de lavoura de arroz.

O experimento I avaliou a competição da cultivar Puitá INTA CL x biótipo de arroz-vermelho suscetível, enquanto o experimento II testou a cultivar Puitá INTA CL x biótipo de arroz-vermelho resistente. Ambos os experimentos foram conduzidos em série de substituição, em que os tratamentos são constituídos por proporçóes de plantas de arroz irrigado e arroz-vermelho. As proporçôes testadas foram: 100:0\% (testemunha arroz irrigado), 75:25, 50:50, 25:75 e $0: 100 \%$ (testemunha arroz-vermelho).

As populaçóes de plantas por vaso foram determinadas em experimentos preliminares, totalizando 24 plantas, equivalente a 942 plantas $\mathrm{m}^{-2}$ (FLECK et al., 2008). A semeadura foi realizada em populaçóes superiores às desejadas em cada tratamento, efetuando desbaste aos sete dias após a emergência (DAE) para retirada das plantas excedentes.

As variáveis área foliar (AF) $\left(\mathrm{cm}^{2}\right.$ planta $\left.^{-1}\right)$ e matéria da parte aérea seca (MPAS) ( g planta $^{-1}$ ) foram analisadas aos 42 DAE. A AF foi determinada com o auxílio de medidor de área foliar (LI 3100C). A MPAS foi quantificada pela pesagem da parte epígea das plantas após serem secas em estufa com circulação forçada de ar a $60^{\circ} \mathrm{C}$ por 72 horas.

Para analisar os dados das variáveis AF e MPAS da planta daninha (arroz-vermelho) e da cultura (arroz irrigado), foi utilizado o método da análise gráfica da produtividade relativa 
(Radosevich, 1987; Roush et al., 1989; Cousens, 1991). Esse procedimento, também chamado de método convencional para experimentos substitutivos, consiste na construção de diagrama com base nas produtividades relativas (PR) e produtividades relativas totais (PRT) nas proporções de plantas de $0,25,50,75$ e $100 \%$ da planta daninha e da cultura. A PR foi calculada pela relaçáo média da mistura/média da monocultura, utilizando-se no cálculo a média por planta de cada espécie em cada unidade experimental.

Nesses diagramas, os resultados obtidos são comparados aos valores da reta hipotética, a qual une os pontos 0 e 100 do diagrama, que representa a ausência de interferência de um genótipo sobre outro. Quando o resultado da PR tende a uma linha reta, isso significa que as habilidades das espécies são equivalentes. Caso a PR resulte em uma linha côncava, indica que existe prejuízo no crescimento de uma ou de ambas as espécies. Ao contrário, se a PR mostrar linha convexa, há benefício no crescimento de uma ou de ambas as espécies.

A PRT foi representada pela soma das produtividades relativas do competidor e da cultura nas respectivas proporçốes de plantas. Se a PRT for igual à unidade (linha reta), significa que ocorre competição pelo(s) mesmo(s) recurso(s); sendo superior a um (linha convexa), náo ocorre competição porque o suprimento de recursos supera a demanda ou porque as espécies possuem diferentes demandas pelo(s) recurso(s) do meio; e, quando inferior a um (linha côncava), significa que ocorre antagonismo, havendo prejuízo mútuo ao crescimento de ambas as espécies.

Os resultados obtidos para AF e MPAS das plantas, expressos em valores médios por planta, foram também submetidos à análise de variância. Quando o teste $\mathrm{F}$ indicou significância $(\mathrm{p} \leq 0,05)$, as médias dos tratamentos foram comparadas pelo teste de Dunnett $(\mathrm{p} \leq 0,05)$, considerando-se os respectivos cultivos isolados como testemunhas.

Além da PR e da PRT, foram calculados índices de competitividade relativa (CR), coeficientes de agrupamento relativo (K) e de competitividade (C). A CR representa o crescimento comparativo da espécie $\mathrm{X}$ em relação à $\mathrm{Y} ; \mathrm{K}$ indica a dominância relativa de uma espécie sobre a outra; $\mathrm{e} C$ aponta qual das espécies é a mais competitiva. Assim, os índices CR, $\mathrm{K}$ e $\mathrm{C}$ indicam qual espécie se manifesta mais competitiva, e sua interpretação conjunta indica com maior segurança a competitividade das espécies (Cousens, 1991). A espécie X é mais competitiva que $\mathrm{Y}$ quando $\mathrm{CR}>1, \mathrm{~K}_{\mathrm{x}}>\mathrm{K}_{\mathrm{y}}$ e $\mathrm{C}>0$; por outro lado, a espécie $\mathrm{Y}$ é mais competitiva que $\mathrm{X}$ quando $\mathrm{CR}<1, \mathrm{~K}_{\mathrm{x}}<\mathrm{K}_{\mathrm{y}}$ e $\mathrm{C}<0$ (Hoffman; Buhler, 2002). Para calcular esses índices, foram usadas as proporçóes 50:50 das espécies, utilizando-se as seguintes equaçóes: $\mathrm{CR}=\mathrm{PR}_{x} / \mathrm{PR}_{y}$; $\mathrm{K}_{\mathrm{x}}=\mathrm{PR}_{\mathrm{x}} /\left(1-\mathrm{PR}_{\mathrm{x}}\right) ; \mathrm{K}_{\mathrm{y}}=\mathrm{PR}_{\mathrm{y}} /\left(1-\mathrm{PR}_{\mathrm{y}}\right) ; \mathrm{C}=\mathrm{PR}_{\mathrm{x}}-\mathrm{PR}_{\mathrm{y}}$ (Cousens; O'NEILL, 1993).

Para analisar estatisticamente a produtividade relativa, primeiramente foi calculada a diferença para os valores de PR (DPR) obtidos nas proporçóes de 25, 50 e 75\% de plantas, em relaçáo aos valores pertencentes às retas hipotéticas obtidas nas respectivas proporçóes. Em seguida, foi utilizado o teste $\mathrm{t}(\mathrm{p} \leq 0,05)$ para testar as diferenças nos índices DPR, PRT, CR, K e C (Roush et al., 1989; Hoffman; Buhler, 2002). As hipóteses de nulidade para testar as diferenças de DPR e C são de que as médias são iguais a $0\left(\mathrm{H}_{0}=0\right)$; para $\mathrm{PRT}$ e $\mathrm{CR}$, que as médias são iguais a $1\left(\mathrm{H}_{0}=1\right)$; para o índice $\mathrm{K}$, que as médias das diferenças entre $\mathrm{K}_{\mathrm{X}}$ e $\mathrm{K}_{\mathrm{Y}}$ são iguais a $0\left[\mathrm{H}_{0}=\left(\mathrm{K}_{\mathrm{X}}-\mathrm{K}_{\mathrm{Y}}\right)=0\right]$.

$\mathrm{O}$ critério para considerar as curvas de PR e PRT diferentes das retas hipotéticas e a existência de diferenças em competitividade pelos índices $\mathrm{CR}, \mathrm{Ke} \mathrm{C}$ foi que, no mínimo em duas proporçóes, ocorressem diferenças significativas pelo teste $t$ (BIANCHI et al., 2006).

\section{RESULTADOS E DISCUSSÃO}

A análise gráfica de PR, para as combinaçōes de plantas do biótipo de arroz-vermelho suscetível (competidor) com a cultivar Puitá INTA CL, para todas as variáveis, demonstrou que os desvios observados em relação às retas esperadas da PR são representados, em geral, por linhas convexas para a planta daninha arroz-vermelho e por linhas côncavas para a cultura (Fig.1), demonstrando que a planta daninha tende a apresentar maior habilidade competitiva. Porém, considerando que para haver significância pelo menos duas proporçôes de plantas devem apresentar diferenças, não foi constatada alteração entre as retas estimadas e as esperadas, tanto para a cultivar Puitá INTA CL quanto para o biótipo suscetível (Tabela 1). Desta forma, pode-se afirmar que os competidores apresentam habilidade competitiva equivalente, ou seja, competem pelo(s) mesmo(s) recurso(s). Creditam-se estes resultados a características intrínsecas dos competidores, uma vez que por pertencerem à mesma espécie, as similaridades na duração do ciclo, estatura, arquitetura do dossel e sistema radicular podem ser responsáveis pela equivalência em crescimento relativo observado.

Com relação à PRT, para as combinaçóes de plantas do biótipo de arroz-vermelho suscetível (competidor) com a cultivar Puitá INTA CL, não houve diferença entre os valores esperados e estimados para as variáveis estudadas, apresentando valores médios próximos à unidade em todas as combinaçôes (Fig. 1 e Tabela 1). Quando os valores de PRT são equivalentes a 1 , correspondendo a uma linha reta, ocorre competiçấo pelos mesmos recursos.

A análise gráfica das combinaçôes de plantas do biótipo de arroz-vermelho resistente e da cultura de arroz cv. Puitá INTA CL, para ambas as variáveis, demonstrou que os desvios observados nas retas $\mathrm{da} P \mathrm{PR}$ em relação às retas esperadas são representados por linhas côncavas nas maiores proporçôes 


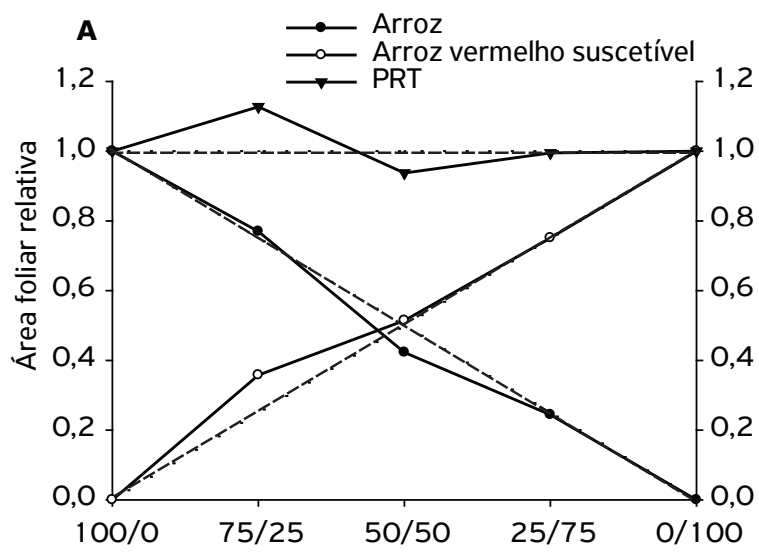

Proporção de plantas arroz/arroz-vermelho suscetível (\%)

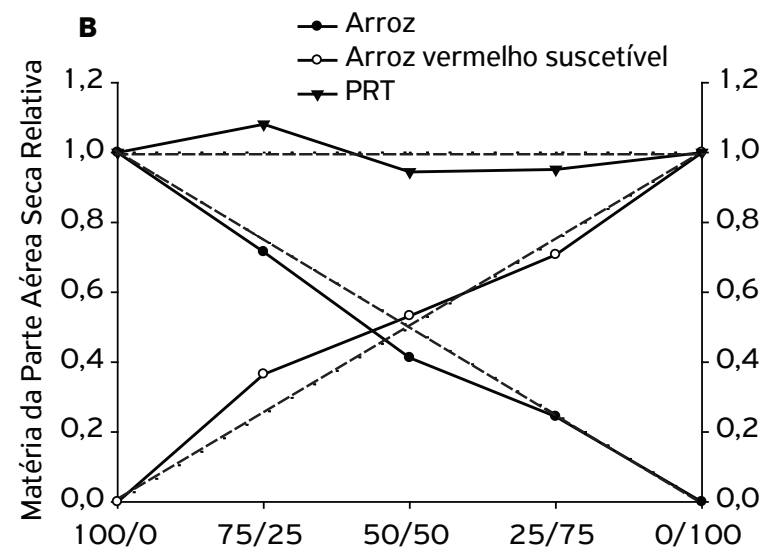

Proporção de plantas arroz/arroz vermelho suscetível (\%)

(०): PR do biótipo suscetível; (•): PR do arroz; ( $\bullet$ ): PRT. Linhas tracejadas referem-se às produtividades relativas hipotéticas, quando não ocorre interferência de uma espécie sobre a outra.

Figura 1. Produtividade relativa (PR) e total (PRT) para área foliar (A) e para matéria da parte aérea seca (B) de plantas de arroz e arroz-vermelho suscetível, FAEM/UFPel, Capão do Leão (RS), 2010/11. da cultura, e convexas na menor (Fig. 2). No caso do competidor ocorre o contrário, ou seja, quando o mesmo está em menores proporçóes, apresenta linha convexa e, quando em maiores proporçóes, linha côncava.

Foram observadas diferenças entre os modelos representados pelas retas estimadas e esperadas apenas para a variável AF do cultivar em competição com o arroz-vermelho, quando este esteve em igual ou menor proporção de plantas; para o arroz-vermelho resistente foram observadas diferenças nas duas variáveis analisadas, quando a planta daninha esteve em menor ou maior proporção de plantas (Tabela 1). Estes resultados demonstram que para o cultivar a competição intraespecífica é mais intensa, ou seja, a cultura prefere ter como vizinha uma planta de arroz-vermelho em relação a uma planta da própria espécie. Já para o arroz-vermelho resistente, independe o vizinho presente no nicho.

Com relação à PRT, foram constatadas diferenças em todas as proporçôes testadas para a variável $\mathrm{AF}$, e não foi observada diferença para a MPAS (Tabela 1). Isso demonstra que os indivíduos interagem pelos mesmos recursos disponíveis no ambiente para o incremento de $\mathrm{AF}$, mas não para a produção de MPAS.

Em todas as proporçóes de plantas do cultivar na associação, para ambas as variáveis estudadas (AF ou MPAS) e competidor (suscetível ou resistente), não foram observadas diferenças em relação ao monocultivo (Tabela 2). Já, em relação aos biótipos de arroz-vermelho (suscetível e resistente), verificou-se aumento das variáveis quando este esteve em menor proporção na associação, enquanto para as demais proporçôes de plantas, em geral, não foram observadas diferenças. Estes resultados permitem inferir que para o arroz-vermelho a competição interespecífica é favorável em relação à competição intraespecífica.

Considerando que a cultura do arroz é mais competitiva que arroz-vermelho quando $\mathrm{CR}>1, \mathrm{~K}_{\mathrm{x}}>\mathrm{K}_{\mathrm{y}}$ e $\mathrm{C}>0$

Tabela 1. Diferenças relativas de produtividade e produtividade relativa total para as variáveis área foliar e matéria da parte aérea seca, nas proporções de plantas 75:25, 50:50 e 25:75 de biótipos de arroz-vermelho suscetível e resistente ao herbicida imazapyr + imazapic, em competição com arroz cultivar Puitá INTA CL.

\begin{tabular}{|c|c|c|c|c|}
\hline & & \multicolumn{3}{|c|}{ Proporção de plantas associadas (arroz:competidor) } \\
\hline & & $75: 25$ & $50: 50$ & $25: 75$ \\
\hline Experimento & Biótipo & \multicolumn{3}{|c|}{ Área foliar } \\
\hline 1 & arroz & $0,02( \pm 0,02)^{\mathrm{ns}}$ & $-0,08( \pm 0,03)^{\mathrm{ns}}$ & $-0,01( \pm 0,03)^{n s}$ \\
\hline 1 & suscetível & $0,11( \pm 0,02)^{*}$ & $0,01( \pm 0,02)^{n s}$ & $0,00( \pm 0,10)^{n s}$ \\
\hline 1 & total & $1,13( \pm 0,04)^{\mathrm{ns}}$ & $0,94( \pm 0,05)^{\mathrm{ns}}$ & $0,99( \pm 0,13)^{n s}$ \\
\hline II & arroz & $-0,03( \pm 0,04)^{\mathrm{ns}}$ & $-0,07( \pm 0,01)^{*}$ & $0,04( \pm 0,01)^{*}$ \\
\hline II & resistente & $0,18( \pm 0,01)^{*}$ & $-0,01( \pm 0,02)^{n s}$ & $-0,14( \pm 0,02)^{*}$ \\
\hline \multirow[t]{2}{*}{ II } & total & $1,15( \pm 0,03)^{*}$ & $0,92( \pm 0,02)^{*}$ & $0,89( \pm 0,02)^{*}$ \\
\hline & & \multicolumn{3}{|c|}{ Matéria da parte aérea seca } \\
\hline I & arroz & $-0,03( \pm 0,03)^{\mathrm{ns}}$ & $-0,09( \pm 0,03)^{\mathrm{ns}}$ & $-0,01( \pm 0,03)^{n s}$ \\
\hline I & suscetível & $0,12( \pm 0,03)^{*}$ & $0,03( \pm 0,02)^{\mathrm{ns}}$ & $-0,04( \pm 0,05)^{n s}$ \\
\hline I & total & $1,08( \pm 0,06)^{\mathrm{ns}}$ & $0,94( \pm 0,04)^{n s}$ & $0,95( \pm 0,07)^{\mathrm{ns}}$ \\
\hline II & arroz & $-0,14( \pm 0,03)^{*}$ & $-0,03( \pm 0,02)^{\mathrm{ns}}$ & $0,03( \pm 0,02)^{\mathrm{ns}}$ \\
\hline II & resistente & $0,12( \pm 0,03)^{*}$ & $0,01( \pm 0,04)^{n s}$ & $-0,16( \pm 0,02)^{*}$ \\
\hline II & total & $0,99( \pm 0,03)^{\mathrm{ns}}$ & $0,98( \pm 0,02)^{\mathrm{ns}}$ & $0,87( \pm 0,02)^{*}$ \\
\hline
\end{tabular}

ns: não significativo; *significativo pelo teste $t(\mathrm{p} \leq 0,05)$. Valores entre parênteses representam os erros padrões das médias. 


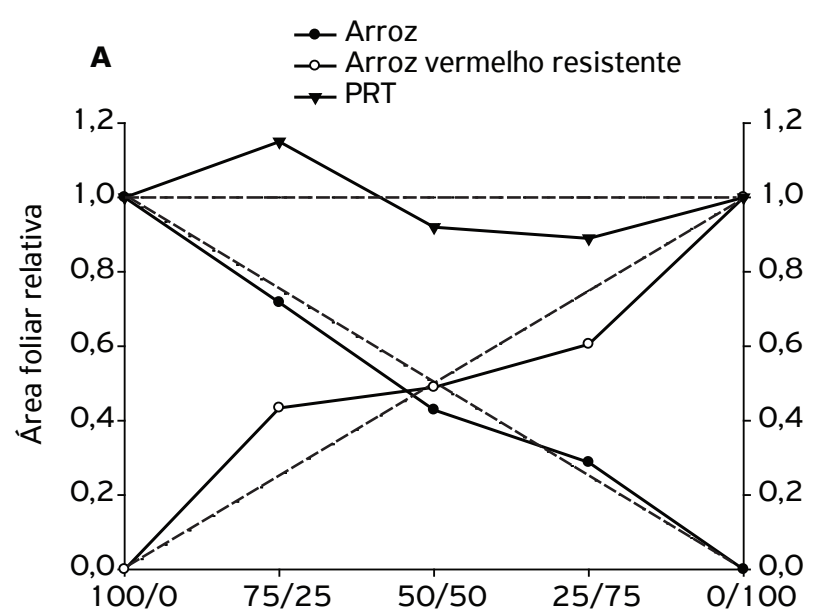

Proporção de plantas arroz/arroz vermelho resistente (\%)

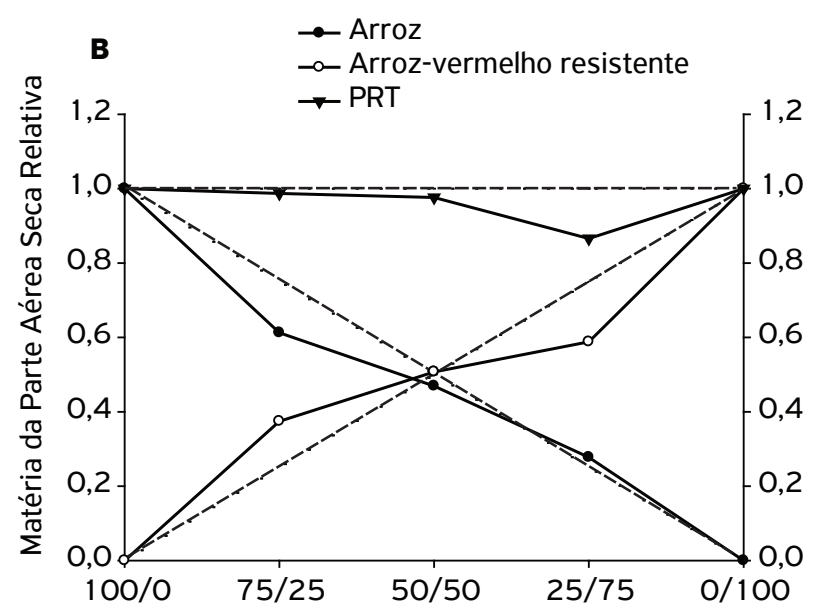

Proporção de plantas arroz/arroz-vermelho resistente(\%)

$(0): P R$ do biótipo resistente; $(\bullet)$ : PR do arroz; ( $\bullet$ ): PRT. Linhas tracejadas referem-se às produtividades relativas hipotéticas, quando não ocorre interferência de uma espécie sobre a outra.

Figura 2. Produtividade relativa (PR) e total (PRT) para área foliar $(A)$ e para matéria da parte aérea seca $(B)$ de plantas de arroz e arroz-vermelho resistente, FAEM/UFPel, Capão do Leão (RS), 2010/11.
(Hoffman; Buhler, 2002) e adotando-se como critério para comprovar superioridade competitiva a ocorrência de diferenças significativas em pelo menos dois índices (BIANCHI et al., 2006), observou-se, para as variáveis avaliadas, que ambos os biótipos possuem competitividade equivalente à cultivar Puitá INTA CL (Tabela 3). Estes resultados corroboraram, em parte, com a análise gráfica. Ainda, pode-se inferir que não houve perda fisiológica para o biótipo resistente devido à tal característica, pois a capacidade competitiva com a cultivar Puitá INTA CL foi equivalente ao observado para o biótipo suscetível.

Causas prováveis para ocorrência da competitividade equivalente entre os biótipos são as semelhanças morfofisiológicas dos biótipos que, por serem da mesma espécie, comportam-se semelhantemente e exploram o mesmo nicho ecológico. Estes resultados vão ao encontro aos observados para populaçóes de E. crusgalli resistentes a clomazone e propanil, (BAgavathiannan et al., 2011), bem como para populaçóes de F. miliacea, resistentes e suscetíveis a ALS (Schaedler, 2011), e Conyza canadensis (DAvis et al., 2009), resistentes e suscetíveis a ALS; inibidores da enzima 5-enolpiruvil-chiquimato-3-fosfato sintetase (EPSPS); e, ALS + EPSPS, em que não houve alteração no crescimento e na competitividade em relação às populações suscetíveis. Ainda, em trabalhos realizados para avaliar a habilidade competitiva de biótipos de Bidens subalternans (LAMEgo et al., 2011) e C. difformis (DAL MAgro et al., 2011) resistente e suscetível aos inibidores da ALS, não foram observadas diferenças, demonstrando que os biótipos são equivalentes em crescimento.

A competição de arroz-vermelho (FLECK et al., 2008) e Echinochloa spp. (AgostinetTo et al., 2008) com a cultura do arroz demonstrou que a cultura foi menos competitiva. Em estudo utilizando cultivar de arroz irrigado EEA 406 como simuladora de arroz-vermelho, não foram observadas diferenças em relação à competição com o cultivar de arroz irrigado IRGA 417 (FLECK et al., 2008), corroborando os resultados encontrados neste experimento. Entretanto, biótipos de C. difformis (DaL Magro et al., 2011) e F. miliacea (SCHAEDLER, 2011) resistentes e suscetíveis

Tabela 2. Resposta de biótipos de arroz-vermelho suscetível e resistente ao herbicida imazapyr + imazapic em competição com arroz cultivar Puitá INTA CL.

\begin{tabular}{|c|c|c|c|c|c|c|c|}
\hline & & \multicolumn{5}{|c|}{ Proporção de plantas } & \multirow{2}{*}{ C.V.(\%) } \\
\hline & & 100:0 & $75: 25$ & $50: 50$ & $25: 75$ & $0: 100$ & \\
\hline Experimento & Biótipo & \multicolumn{6}{|c|}{ Área foliar } \\
\hline 1 & arroz & 205,4 & $210,7^{\text {ns }}$ & $173,7^{\text {ns }}$ & $200,4^{\text {ns }}$ & - & 14,7 \\
\hline 1 & suscetível & - & $429,3^{*}$ & $308,7^{\text {ns }}$ & $300,4^{\text {ns }}$ & 300,1 & 15,9 \\
\hline II & arroz & 225,0 & $215,3^{\text {ns }}$ & $192,3^{\text {ns }}$ & $258,9^{\text {ns }}$ & - & 8,9 \\
\hline \multirow[t]{2}{*}{ II } & resistente & - & $479,3^{*}$ & $270,3^{\text {ns }}$ & $222,8^{*}$ & 276,1 & 6,1 \\
\hline & & \multicolumn{6}{|c|}{ Matéria da parte aérea seca } \\
\hline I & arroz & 1,4 & $1,3^{\text {ns }}$ & $1,1^{\mathrm{ns}}$ & $1,3^{\text {ns }}$ & - & 17,1 \\
\hline 1 & suscetível & - & $2,7^{*}$ & $1,9^{\text {ns }}$ & $1,7^{\mathrm{ns}}$ & 1,8 & 14,1 \\
\hline II & arroz & 1,5 & $1,3^{\mathrm{ns}}$ & $1,4^{\mathrm{ns}}$ & $1,7^{\mathrm{ns}}$ & - & 10,2 \\
\hline II & resistente & - & $2,9^{*}$ & $2,0^{\text {ns }}$ & $1,5^{\mathrm{ns}}$ & 1,9 & 13,3 \\
\hline
\end{tabular}

ns: não significativo; *significativo em relação à respectiva testemunha (100\%) pelo teste de Dunnett $(p \leq 0,05)$. 
Tabela 3. Índices de competitividade entre biótipos de arroz-vermelho suscetível e resistente ao herbicida imazapyr + imazapic em competição com arroz cultivar Puitá INTA CL, expressos por competitividade relativa (CR) e coeficientes de agrupamento relativo (K) e de competitividade (C).

\begin{tabular}{|c|c|c|c|c|}
\hline & CR & Kx (arroz) & Ky (suscet. ou resist.) & C \\
\hline \multicolumn{5}{|c|}{ Experimento I - arroz: suscetível } \\
\hline $\mathrm{AF}$ & $0,82( \pm 0,05)^{n s}$ & $0,78( \pm 0,14)^{\mathrm{ns}}$ & $1,01( \pm 0,12)$ & $-0,08( \pm 0,03)^{n s}$ \\
\hline MPAS & $0,84( \pm 0,07)^{n s}$ & $0,81( \pm 0,12)^{\mathrm{ns}}$ & $0,96( \pm 0,07)$ & $-0,12( \pm 0,03)^{*}$ \\
\hline \multicolumn{5}{|c|}{ Experimento II - arroz: resistente } \\
\hline $\mathrm{AF}$ & $0,88( \pm 0,04)^{n s}$ & $0,75( \pm 0,01)^{*}$ & $0,97( \pm 0,08)$ & $-0,06( \pm 0,02)^{n s}$ \\
\hline MPAS & $0,95( \pm 0,11)^{n s}$ & $0,89( \pm 0,08)^{\mathrm{ns}}$ & $1,06( \pm 0,14)$ & $-0,04( \pm 0,05)^{n s}$ \\
\hline
\end{tabular}

AF: área foliar; MPAS: matéria da parte aérea seca; ns: não significativo; *significativo pelo teste $t(p \leq 0,05)$. Valores entre parênteses representam os erros padrões das médias.

apresentaram menor habilidade competitiva em comparação à cultura do arroz irrigado.

Mesmo em condições não competitivas, a inibição da ALS não influencia o aparato fotossintético dos biótipos resistentes, ou seja, não ocasiona o desempenho ecológico inferior destes em relação aos suscetíveis (RIzZARDi et al., 2002), como observado para a variável matéria da parte aérea de Kochia scoparia, resistente e suscetível aos inibidores de ALS (Thompson et al., 1994; Christoffoleti et al., 1997). Resultados semelhantes também foram observados em Euphorbia heterophylla (TREZZI; Vidal, 2000; Brighenti et al., 2001) e Bidens subalternans (LAMEgo et al., 2011).

Estudos relacionados à competitividade do arroz cultivado e às diferentes populaçóes de arroz-vermelho ainda são limitados. O conhecimento da dinâmica e da competitividade entre plantas, em especial o arroz irrigado e o arroz-vermelho, têm importância para a tomada de decisão de controle da planta daninha, contribuindo para a reduçáo do uso de herbicidas na cultura do arroz irrigado.

\section{CONCLUSÃO}

Para o arroz-vermelho suscetível ou resistente ao herbicida, a competição interespecífica é favorável em relação à competição intraespecífica, enquanto para a cultura do arroz irrigado independe o competidor.

A cultivar Puitá INTA CL, em geral, apresenta habilidade competitiva equivalente aos biótipos de arroz-vermelho suscetível ou resistente ao herbicida imazapyr + imazapic, competindo pelos mesmos recursos do ambiente.

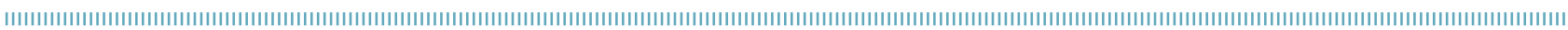

\section{REFERÊNCIAS}

AGOSTINETTO, D.; FLECK, N.G.; RIZZARDI, M.A.; MEROTTO JR, A.; VIDAL, R.A. Arroz vermelho: ecofisiologia e estratégias de controle. Ciência Rural, v.31, n.2, p.341-349, 2001.

AGOSTINETTO, D.; GALON, L.; MORAES, P.V.D.; RIGOLI, R.P.; TIRONI, S.P.; PANOZZO, L.E. Competitividade relativa entre cultivares de arroz irrigado e biótipo de capim-arroz (Echinochloa spp.). Planta Daninha, v.26, n.4, p.757-766, 2008.

BAGAVATHIANNAN, M.V.; NORSWORTHY, J.K; JHA, P.; SMITH, K. Does resistance to propanil or clomazone alter the growth and competitive abilities of barnyardgrass (Echinochloa crus-galli)? Weed Science, v.59, n.3, p.353-358, 2011.

BIANCHI, M.A.; FLECK, N.G.; LAMEGO, F.P. Proporção entre plantas de soja e plantas competidoras e as relações de interferência mútua. Ciência Rural, v.36, n.5, p.1380-1387, 2006.

BRIGHENTI, A.M.; GAZZIERO, D.L.P.; VOLL, E.; ADEGAS, F.S.; VAL, W.M.C. Análise de crescimento de biótipos de amendoimbravo (Euphorbia heterophylla) resistente e suscetível aos herbicidas inibidores da ALS. Planta Daninha, v.19, n.1, p.51-59, 2001.
CHRISTOFFOLETI, P.J.; WESTRA, P.; MOORE, F. Growth analysis of sulfonylurea-resistant and susceptible kochia (Kochia scoparia). Weed Science, v.45, n.5, p.691-695, 1997.

CHRISTOFFOLETI, P.J.; LÓPEZ-OVEJERO, R.F.; NICOLAI, M.; VARGAS, L.; CARVALHO, S.J.P.; CATANEO, A.C.; CARVALHO, J.C.; MOREIRA, M.S. Aspectos de resistência de plantas daninhas a herbicidas. Piracicaba: (HRAC-BR), 2008. 120p.

CONAB. Arroz-Brasil. Série Histórica de áreas, produtividade e produção. Disponível em: www.conab.gov.br. Acesso em: 18 nov. 2011.

COUSENS, R. Aspects of the design and interpretation of competition (interference) experiments. Weed Technology, v.5, n.3, p.664-673, 1991.

COUSENS, R.; O'NEILL, M. Density dependence of replacement series experiments. Oikos, v.66, n.2, p.347-352, 1993.

DAL MAGRO, T.; SCHAEDLER, C.E.; FONTANA, L.C.; AGOSTINETTO, D.; VARGAS, L. Habilidade competitiva entre biótipos de Cyperus difformis $L$. resistente ou suscetível a herbicidas inibidores de ALS e destes com arroz irrigado. Bragantia, v.70, n.2, p.294-301, 2011. 
DAVIS, V.M.; KRUGER, G.R.; STACHLER, J.M.; LOUX, M.M.; JOHNSON, W.G. Growth and seed production of horseweed (Conyza canadensis) populations resistant to glyphosate, als-inhibiting, and multiple (glyphosate + als-inhibiting) herbicides. Weed Science, v.57, n.5, p.494-504, 2009.

FISCHER, A.J.; RAMIREZ, A. Red rice (Oryza sativa): competition studies for management decisions. International Journal of Pest Management, v.39, n.2, p.133-138, 1993.

FLECK, N.G.; AGOSTINETTO, D.; RIZZARDI, M.A.; BIANCHI, M.A.; MENEZES, V.G. Interferência de plantas concorrentes em arroz irrigado modificada por métodos culturais. Planta Daninha, v.22, n. 1, p. 19-28, 2004.

FLECK, N.G.; AGOSTINETTO, D.; GALON, L.; SCHAEDLER, C.E. Competitividade relativa entre cultivares de arroz irrigado e biótipo de arroz-vermelho. Planta Daninha, v.26, n.1, p. 101-111, 2008.

HOFFMAN, M.L.; BUHLER, D.D. Utilizing Sorghum as functional model of crop-weed competition. I. Establishing a competitive hierarchy. Weed Science, v.50, n.4, p.466-472, 2002.

LAMEGO, F.P.; VIDAL, R.A.; BURGOS, N.R. Competitiveness of ALS inhibitors resistant and susceptible biotypes of greater beggarticks (Bidens subalternans). Planta Daninha, v.29, n.2, p.457-464, 2011.

RADOSEVICH, S.; HOLT, J.; GHERSA, C. Weed ecology: implications for vegetation management. 2. ed. Wiley, 1997.589p.
RADOSEVICH, S.R. Methods to study interactions among crops and weeds. Weed Technology, v.1, n.3, p.190-198, 1987.

RIZZARDI, M.A.; VIDAL, R.A.; FLECK, N.G.; AGOSTINETTO, D. Resistência de plantas aos herbicidas inibidores da acetolactato sintase. Planta Daninha, v.20, n. 1, p.149-158, 2002.

ROUSH, M.L.; RADOSEVICH, S.R.; WAGNER, R.G.; MAXWELL, B.D.; PETERSEN, T.D. A comparison of methods for measuring effects of density and proportion in plant competition experiments. Weed Science, v.37, n.2, p.268-275, 1989.

SCHAEDLER, C.E. Resistência de Fimbristylis miliacea (L.) Vahl aos herbicidas inibidores da enzima ALS. 2011 . 126f. Tese (Doutorado) Faculdade de Agronomia "Eliseu Maciel", Universidade Federal de Pelotas, Pelotas, 2011.

THOMPSON, C.R.; THILL, D.C.; SHAFII, B. Growth and competitiveness of sulfonylurea-resistant and susceptible kochia (Kochia scoparia). Weed Science, v.42, n.2, p.172-179, 1994.

TREZZI, M.M.; VIDAL, R.A. Crescimento estival de três biótipos de leiteira resistentes e um suscetível aos herbicidas inibidores da acetolactato sintase. Revista Brasileira de Herbicidas, v. 1, n.3, p.255-260, 2000.

VIDAL, R.A.; MEROTTO JR, A. Herbicidologia. Porto Alegre: Evangraf, 152p., 2001.

VILA-AIUB, M.M.; NEVE, P.; POWLES, S.B. Fitness costs associated with evolved herbicide resistance alleles in plants. New Phytologist, v. 184, n.4, p.751-767, 2009. 